\title{
Heterogeneous Backhaul for Cloud-Based Mobile Networks
}

\author{
Jens Bartelt, Gerhard Fettweis \\ Vodafone Chair Mobile Communications Systems \\ Dresden University of Technology, Germany \\ \{jens.bartelt, fettweis\}@ifn.et.tu-dresden.de
}

\author{
Dirk Wübben \\ Dep. of Communications Engineering \\ University of Bremen, Germany \\ wuebben@ant.uni-bremen.de
}

\author{
Mauro Boldi, Bruno Melis \\ Telecom Italia, Turin, Italy \\ \{mauro.boldi, bruno1.melis \\ @ telecomitalia.it
}

\begin{abstract}
To meet the increasing capacity demands of future mobile networks, dense deployment of radio access nodes in combination with partly centralized processing by means of a cloudbased architecture is a promising option. In such an architecture, the design and optimization of the backhaul plays a crucial role. In this paper, we review different backhaul technologies available and discuss their characteristics for use in cloud-based networks. We point out how a heterogeneous backhaul network and a flexible centralization enables the proposed architecture and give an outlook on how a joint design of access and backhaul can help in meeting the increased demands.
\end{abstract}

Index Terms-heterogeneous backhaul, centralized processing, C-RAN

\section{INTRODUCTION}

Future mobile networks will have to provide an exceptionally high traffic volume with diverse data rates from machineto-machine (low data rates) to 3D applications (high data rates). To cope with the exponentially growing traffic demand, small cells appear to be a relevant option for mobile network evolution. As the radio access point is placed closer to the user, the same spectrum could be reused to increase the overall capacity of the network tremendously. Furthermore, as the users are usually in the range of several small cells, the transmit and receive signals should be processed jointly. However, the successful dense deployment of small cells requires smart access and interference management techniques.

Centralised processing permits the implementation of efficient interference avoidance and cancelation algorithms across multiple cells [1], [2]. It provides the means to selectively turn radio access points on and off in order to balance traffic in scenarios that have high traffic fluctuations. Cloud-RAN (CRAN) has recently attracted a great deal of attention as one possible way to efficiently centralise computational resources, to balance throughput fluctuations, and to implement intercell coordination. In C-RAN, multiple sites are connected to a central data centre where all baseband processing is performed. Transmitted and received radio signals are exchanged over fibre transmission lines between Remote Radio Heads (RRHs) and the data centre. At present, only fibre-links are capable of supporting the data rates required, e.g., of about $10 \mathrm{Gbps}$ for TD-LTE with a $20 \mathrm{MHz}$ bandwidth. This constitutes the main drawback of C-RAN which is the need for a link that supports very high data rates. Due to the use of optical fibre, C-RAN deployments are less flexible as only spots with existing fibre-access may be chosen or fibre-access must be deployed, which is very expensive. Hence, there is a tradeoff between centralised processing requiring high-capacity backhaul links, and de-centralised processing which requires traditional backhaul to transport the user- and control-data to and from the base station (BS).

This trade-off can be exploited by the novel concept "Radio Access Network-as-a-Service" (RANaaS) proposed by the iJOIN project [3], [4]. Relying on the progress in cloud computing, the RANaaS concept allows classical functionalities usually processed within a small cell to be partially (or fully) offloaded to a cloud platform, in order to benefit, not only from computing power, but also from centralisation (coordination with other small cells). Obviously, this new paradigm is highly dependent on the RANaaS platform resource availability and also on the backhaul properties. Thus, particular attention is given to a joint design of the radio access and the backhaul. The level of functional split in the RANaaS platform naturally takes into account the backhaul limitations, while other optimisations may rely on the introduction of (local) network controller collecting inputs from small cells and the backhaul nodes to enhance network management. Within this paper, we discuss the properties of possible backhaul approaches for the network architecture envisioned and have, therefore, organized the rest of the paper as follows: in Sec. II, we give an overview over the system architecture envisioned and in Sec. III, we analyse different backhaul technologies available. Sec. IV then describes the specific challenges that cloud based networks impose on the backhaul network and gives examples of how to jointly optimize access and backhaul, before our conclusions are given in Sec. V.

\section{SySTEM ARCHITECTURE}

Figure 1 shows the envisaged architecture of the iJOIN project for the RANaaS concept. Based on a dense deployment, several small cells (iSCs) are in the range of one user equipment (UE), such that a joint processing of the received and transmitted signals is favourable. This requires an adopted network architecture, allowing for an efficient implementation with a multitude of spatially distributed nodes, a split of PHY functionality in the access network and centralised RAN processing. Thus, the boundaries between the access network itself and a heterogeneous backhaul are blurred and a joint 


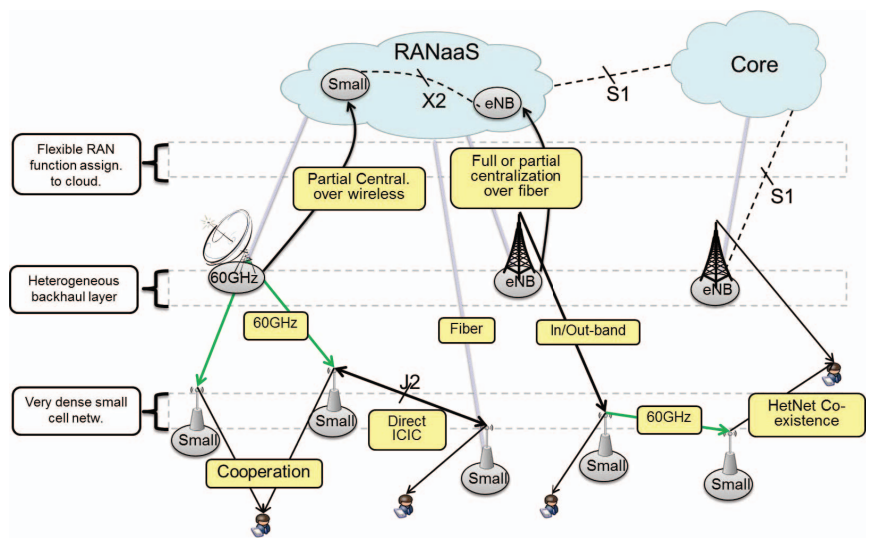

Fig. 1. Mobile network architecture for a RANaaS implementation.

design of both becomes necessary. In order to develop such a new architecture, physical layer technologies which are capable to provide the required data rates and which can be flexibly operated based on backhaul layer parameters are of interest. Promising PHY techniques for partly/fully decentralized processing that are able to changing backhaul and access layer parameters are, e.g., Cooperative Multi-Point (CoMP) that processes jointly transmitted and received user signals and distributed estimation algorithms such as distributed turbo processing (DTP) and In-Network-Processing (INP) [3].

\section{BACKHAUL TECHNOLOGIES}

This section gives an overview of popular wired and wireless backhaul technologies.

\section{A. Wired Backhaul}

For wired backhauling, either copper or fiber are the applied physical media.

1) Copper: With regards to copper based backhauling, one possible approach is to utilize the commonly available xDSL (Digital Subscriber Line) access. The letter ' $x$ ' of the acronym represents the many different varieties of DSL (e.g. different modulation techniques). xDSL evolution, over the years, has been driven by the goal of providing increased bandwidth while leveraging the existing copper of the fixed telephone infrastructure. On the lower end of the xDSL technology, there is ADSL (Asymmetric DSL), while at the high end there is the VDSL2 (Very high speed DSL2) that is suitable for very high bit rate connections over short distances (e.g., connections between/within buildings) [5]. Table I provides a synopsis of the xDSL technology evolution and indicates the maximum downstream and upstream data rate achievable, in the absence of crosstalk and for a very short loop length (i.e., back to back).

ADSL2+ utilizes $2.2 \mathrm{MHz}$ of bandwidth and is limited to speeds of approximately $24 \mathrm{Mbps}$ downstream and 1 Mbps upstream, whereas VDSL2 utilizes up to $30 \mathrm{MHz}$ of bandwidth to provide speeds of $100 \mathrm{Mbps}$ both downstream and upstream within 300-400 m. Rates well above $100 \mathrm{Mbps}$ can be achieved for VDSL through line bonding, while data
TABLE I

EVOLUTION OF XDSL TECHNOLOGY

\begin{tabular}{l|l|l|l} 
Technology & ITU Rec. & $\begin{array}{l}\text { Max. Down- } \\
\text { stream }\end{array}$ & $\begin{array}{l}\text { Max. } \\
\text { Upstream }\end{array}$ \\
\hline \hline ADSL & G.992.1 & $8 \mathrm{Mbps}$ & $1 \mathrm{Mbps}$ \\
\hline ADSL2 & G.992.3/4 & $12 \mathrm{Mbps}$ & $1 \mathrm{Mbps}$ \\
\hline ADSL2+ & G.992.5 & $24 \mathrm{Mbps}$ & $1 \mathrm{Mbps}$ \\
\hline VDSL & G.993.1 & $52 \mathrm{Mbps}$ & $16 \mathrm{Mbps}$ \\
\hline VDSL2 17a & G.993.2 & $100 \mathrm{Mbps}$ & $50 \mathrm{Mbps}$ \\
\hline VDSL2 30a & G.993.2 & $100 \mathrm{Mbps}$ & $100 \mathrm{Mbps}$
\end{tabular}

rate reduction caused by crosstalk can be compensated through the cancellation technique called "vectoring".

Based on Discrete MultiTone (DMT) line code, the VDSL2 standard specifies several profiles, optimized for different deployment scenarios. A significant feature is also that VDSL2 uses Ethernet as a multiplexing technology, eliminating the use of Asynchronous Transfer Mode (ATM).

The main advantage of XDSL is speed of deployment, thanks to the existing and widespread fixed telephone infrastructure, counterbalanced by high blocking probability, lack of fault management tools and insufficient bandwidth to support the high data rates provided even by today's wireless communication systems.

2) Fiber: The alternative to copper for mobile backhaul are fiber based solutions that can provide almost unlimited capacity. The main fiber access options include GPON (Gigabit Passive Optical Network), Carrier Ethernet, and point-to-point (PTP) fiber. A passive optical network (PON) is a point-tomultipoint fiber based network architecture in which passive optical splitters are used to enable a single optical fiber to serve multiple termination points, as shown in Figure 2. A PON consists of an Optical Line Termination (OLT) at the service provider central office and a number of Optical Network Units (ONUs) at the network termination point (e.g. cabinet curb, building, home).

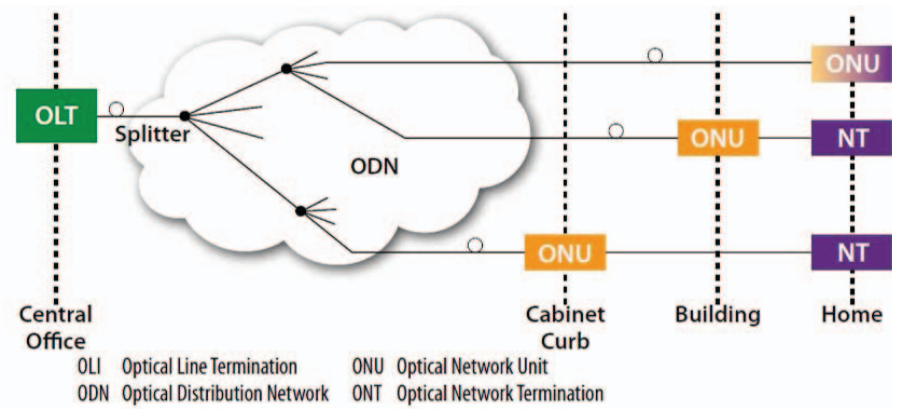

Fig. 2. General structure of a GPON network

In a mixed residential and mobile backhaul scenario, some ONUs support residential or business users, while others serve mobile base transceiver stations (called BTS or eNodeB, depending on the technology) [6]. Downstream signals are broadcast to all users, which share the same fiber (encryption ensures confidentiality of data), while upstream signals are 
combined using a multiple access protocol, usually Time Division Multiple Access (TDMA). When referring to TDMA PON, there are two prominent standards that can be used: GPON [7] and EPON [8]. The standards permit several choices of bit rate, but the industry has converged on 2.488 Gbit/s of downstream bandwidth and $1.244 \mathrm{Gbit} / \mathrm{s}$ of upstream bandwidth.

The next step in GPON evolution has been taken within the FSAN/ITU-T (Full Service Access Network) standardization group [9] with the definition of the Next Generation PON (also known as XG-PON) that may enable a downstream bandwidth of $10 \mathrm{Gbit} / \mathrm{s}$, upstream bandwidth of $2.5 \mathrm{Gbit} / \mathrm{s}$, maximum splitting factor of 128 and maximum range of $20 \mathrm{~km}$ [10]. A more enhanced generation of GPON networks, denoted as NG-GPON2, is also being standardized within the FSAN/ITU-T group. The NG-PON2 has the following performance specifications: bandwidth of $40 \mathrm{Gbit} / \mathrm{s}$ and 10 Gbit/s for the downstream and upstream respectively, splitting factor from 64 to 1000 and range up to $40 \mathrm{~km}$.

Finally, recent innovations in Ethernet networking technology have progressively enhanced both the scalability and capability of Ethernet as a carrier-grade transport technology for mobile backhauling. Gigabit Ethernet (GbE) builds on top of the Ethernet protocol, but increases speed tenfold over Fast Ethernet to $1 \mathrm{Gbit} / \mathrm{s}$. This protocol, which was standardized in June 1998, promises to be a dominant player in high-speed local area network backbones. In 2007, the protocol has been extended to $10 \mathrm{Gbit} / \mathrm{s}(10 \mathrm{GbE})$ and in 2010, the standard for $100 \mathrm{GbE}$ was approved, which specifies the transmission of $100 \mathrm{Gbit} / \mathrm{s}$ via 4 wavelength channels of $25 \mathrm{Gbit} / \mathrm{s}$ each.

\section{B. Wireless Backhaul}

Wireless backhaul can be differentiated by the frequency bands being used. Due to the different channel characteristics in the different bands, each technology has its own advantages and disadvantages. However, the aspects they have in common are that they are faster and cheaper to deploy than fiber, as expensive earthworks are not required. So far, the following technologies have been considered:

1) Free Space Optics: Similar to fiber, free space optics (FSO) uses light to transmit data, but instead of relying on fiber as a transmission medium, it uses free space propagation. FSO offers high data rates of multiple Gbps due to the availability of very high bandwidths and usually does not have to be licensed, further lowering cost and deployment time. However, it suffers heavily during snowfall and fog, limiting either range (up to a few $\mathrm{km}$ ) or availability (depending on geographic location) [11]. Due to the very narrow beamwidth, FSO links create nearly no interference between each other, but they also have to be carefully aligned and are susceptible to thermal expansion, building sway, and vibration. When they are facing east to west, they can also suffer from sunlight effects [12].

2) Relaying: Backhaul technologies that operate in the same frequency band as the access link (LTE or WiFi) are commonly referred to as relaying. It is an inherent advantage that relays use the same transmission technology and licenses as the access link. However, they also have the same limitations in terms of range (up to a few $\mathrm{km}$ ), capacity (a few hundred Mbps), and interference. Furthermore, depending on the carrier allocation, relays either introduce more interference into the network, or reduce the bandwidth available for the access links.

The $5 \mathrm{GHz}$ band is also used by many users, as it is specified as a WiFi band. This increases interference, which further limits data rates and decreases availability. The relaying systems are also particular vulnerable to interception, because all other wireless systems use highly directive beams that would require an interceptor be suspiciously deployed in the connection's line-of-sight (LOS).

3) Microwave: Traditional microwave systems use carrier frequencies between $6 \mathrm{GHz}$ and $42 \mathrm{GHz}$, can offer data rates between a few hundred Mbps and $1 \mathrm{Gbps}$, and use licensed spectrum, which increases costs and deployment time. Due to moderate pathloss, very high ranges on the order of tens of $\mathrm{km}$ can be achieved when used with highly directive antennas [13], [14].

4) mmWave: The so-called millimeter-wave (mmWave) technology has only recently begun to appear due to advances in circuit technologies, and currently offers three bands at 60, 70/80, and $90 \mathrm{GHz}$. Depending on the geographical area, each band offers up to $9 \mathrm{GHz}$ spectrum, enabling multi-Gbps data rates. As the $60 \mathrm{GHz}$ band is unlicensed and the higher bands only require an easy and inexpensive licensing process, the links can be deployed much faster and at lower cost. However, the pathloss at theses frequencies is very high (30 $\mathrm{dB}$ more at $60 \mathrm{GHz}$ than at $2 \mathrm{GHz}$ ) and the signals are attenuated even further by atmospheric absorption and rain. To compensate for the pathloss, either the transmission power has to be increased, which is limited by regulations and hardware constraints, or very highly directional antennas have to be deployed. Commercial products (e.g., [15]) use antennas with a beamwidth of less than one degree and a gain of up to $51 \mathrm{dBi}$. Still, the range is limited to $10 \mathrm{~km}$ at a data rate of $1 \mathrm{Gbps}$.

On the other hand, high signal attenuation has the advantage that interference between mmWave links is very low and that directive antennas increase security against eavesdropping. However, the small beamwidth limits multipath effects, making spatial diversity multiplexing techniques (MIMO) more difficult and requires LOS. Yet, even without MIMO, it has been shown in experimental demonstrators that at smaller distances mmWaves can achieve data rates of up to $10 \mathrm{Gbps}$ [16], closing the gap between wireless and fiber backhaul. This makes mmWave links a viable option for backhaul on the "last mile" towards the base stations.

An overview of the approximate maximum data rates and ranges of the different backhaul technologies is given in Fig. 3. The given numbers can only serve as a rough comparison, as the actual performance varies greatly, and it should be kept in mind that range and data rate directly depend on each other; the higher the range, the lower the rate 
that can be achieved. The range of every technology can be easily extended by a serial concatenation of multiple links in a multi-hop manner.

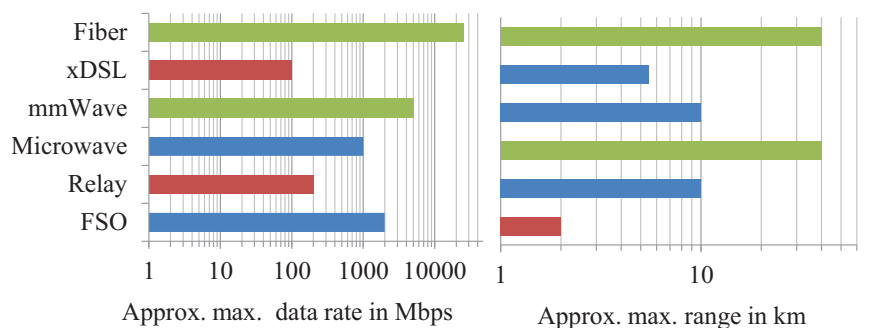

Fig. 3. Overview over max. data rate and range of different backhaul technologies.

\section{Heterogeneous Backhaul for Cloud-Based MOBILE NETWORKS}

\section{A. Backhaul as an Enabler of Cloud-based Mobile Networks}

The backhaul in a cloud based heterogeneous network architecture has to be capable of enabling different types and degrees of centralized processing. The challenges for designing and optimizing the backhaul are threefold:

1) Heterogeneity: The large number of iSCs and their diverse deployment options require heterogeneous backhaul, because not all technologies discussed in Section III are suitable for every deployments. Fiber provides the largest data rates at the largest distances and is therefore, in principle,the technology of choice. However, it might not be implementable for specific links due to geographical or economic reasons. The xDSL technology is a suitable candidate for iSCs that are deployed by shops or companies for indoor coverage, as XDSL access is usually already available.

FSO and mmWave links can cover the "last mile" towards iSCs deployed in large quantities at sites like lamp posts or billboards, if fiber is too expensive. They can also be used to cross obstacles like rivers or large roads, where fiber cannot be deployed because of safety or ownership issues. Multihop concatenation of those links can increase their range or overcome non-line-of-sight (NLOS) paths at the cost of additional latency. For example, the data of iSCs deployed along a street can be transported by mmWave links from one to the other in a multi-hop manner to a transport node with fiber access to the RANaaS. However, in such multihop transmissions, the data to be backhauled multiplies with the number of hops, which increases the bandwidth required towards the last link. Inter-site connections are also a viable use case for mmWave technology, as the typical inter-site distance of small cells is well within the range of mmWave technology.

2) Data Rate: If fully centralized processing in the RANaaS is envisioned, a huge amount of data would have to be transported by the backhaul between the RANaaS and the iSCs. If only a part of the processing is shifted to the RANaaS, the amount of data depends on the particular shift of functionality. For example, streaming of raw I/Q data from an iSC to the RANaaS can easily require ten times the capacity required for transmitting user data. As the backhaul capacity can also be subject to changes (e.g., wireless backhaul), approaches allowing for a flexible functional split that can be adapted to the available backhaul properties are required.

3) Latency: As in the network architecture envisioned processing might take place geographically far away from the user, latency is a key constraint on the backhaul. In [17], requirements for the one way latency in small cell backhaul are defined that range between $2 \mathrm{~ms}$ (ideal) to $60 \mathrm{~ms}$ (nonideal), which shall serve as a comparison in the following. The latency can be attributed to three separate contributions: propagation time, processing duration, and protocol latency.

- Propagation: It is well known that wireless signals travel through air roughly at the speed of light, i.e., at $c \approx 3 \cdot 10^{8}$ $\mathrm{m} / \mathrm{s}$. Depending on the material, the propagation speed is slower in fiber and copper cables, with a speed between $66 \%$ and $95 \%$ of $c$. If we assume that the distance between the user and the actual processing in the cloud is $100 \mathrm{~km}$, the propagation time at $66 \%$ of $c$ is $0.5 \mathrm{~ms}$, which is already close to the order of magnitude of the total tolerable delay.

- Processing: At each node in the network the data is processed to a certain degree. The total latency therefore depends on the number of hops in the end-to-end link, and the processing time at each node depends on the amount of processing and the hardware deployed. Currently, decoding is often the most time consuming process on lower layers, due to the iterative nature of turbo decoding. For example in [16], the encoding for a $60 \mathrm{GHz}$ transmission introduces a delay of $0.99 \mu \mathrm{s}$, while the decoding accounts for $2.59 \mu \mathrm{s}$. In [18], a total delay of $>40 \mu \mathrm{s}$ is given for a $70 \mathrm{GHz}$ wireless link.

- Protocol: A multitude of protocols on different layers influence the latency of the transmission as well. For example, in any frame-based transmission, usually a data bit can only be processed once the complete frame is received. In such a case, the frame length and bit rate influence the delay, and should be carefully adapted for each link. Time duplex or multiplex are additional examples of how protocols introduce delay.

The latency in cloud-based networks scales with the distance between the UE and the RANaaS, the number of nodes in the link, and the amount of processing at each node. The propagation time should be reduced by carefully choosing the geographical location of the RANaaS hardware. Processing and protocol latency can be reduced by keeping the processing at each node to a minimum, especially if a multi-hop connection is utilized.

Altogether, the amount of processing that can be centralized has to take the actual deployment into account. Multi-hop links might be too slow for PHY processing that requires accurate channel state information and receive signals. However, functions such as RRM or QoS control that are less time critical 
are still viable centralization options, even for higher latencies.

\section{B. Joint Access and Backhaul Design and Energy Efficient Networks}

To overcome the challenges presented, we envision a flexible split of the functionality between iSCs and the RANaaS platform to adapt to different amounts of centralized processing, as well as varying backhaul capacities. This approach combines the advantages of C-RAN, where almost no processing takes place at RRHs, and the conventional approach, where a great amount of processing takes place at BSs.

One example for shifting processing from iSCs towards the RANaaS is to utilize the RRH approach not only for fiber, but also for wireless technologies. Usually, while using wireless backhaul, uplink user data would have to be decoded at the iSC to reduce the bandwidth required and then re-encoded for transmission towards the RANaaS. With the large amount of bandwidth available for mmWave backhaul, the idea is to skip decoding at the iSC and forward sampled receive symbols for decoding at the RANaaS directly. To reduce the amount of data to be relayed, compression techniques can be employed. If I/Q data is oversampled or is received from multiple antennas, the samples are correlated and can be compressed if the correlation is exploited carefully. Similar to the RRH approach, this scheme has the benefit that the iSCs require less hardware, which decreases the deployment cost as well as the energy consumption and latency.

An opposite example for shifting processing towards the iSCs is the so-called frequency domain Radio-over-Fiber (RoF) approach for the downlink. In a conventional RoF architecture, time domain signals that include the superimposed signals for multiple users as well as overhead, such as the cyclic prefix and guard carriers, are transmitted from the central unit to the iSCs. By relocating the FFT/IFFT functionality to the iSCs, the data can be transmitted in the frequency domain between the RANaaS and the iSC, reducing the overhead and therefore the required capacity.

Besides, the conventional RoF architecture is highly inefficient when it is necessary to perform signal processing operations on a per-user basis, like adaptive beamforming or coordinated transmission between multiple iSCs. For these techniques, the weight factors for each user are different, and have to be continuously updated in order to track the movement of the user within the cell and track the channel variations. The application of these algorithms in a conventional $\mathrm{RoF}$ architecture requires performing all the signal processing operations in the RANaaS prior to the IFFT operation, where the signals of different users are still available separately. This architecture lacks flexibility because it is necessary to transmit one composite signal for each antenna element of each iSC. It follows, that as the number of iSCs connected via one link increases, the transmission capacity available on the fiber rapidly becomes a bottleneck. In contrast, using frequency domain RoF, the data has to be transmitted only once on a per-user basis and can be separately weighted at each antenna element.

\section{Conclusions}

In this paper, we analysed the challenges for backhaul networks arising from a cloud-based architecture. We compared different backhaul technologies and concluded that only a heterogeneous mix can enable the envisioned architecture. Fiber and mmWave seem to be the most promising technologies and will be investigated more closely in our future work. Depending on the degree of centralization, the rate demands and the latency requirements for the backhaul increase. Thus, a flexibel functional split and a joint optimization of access and backhaul, as we outlined by the examples of the mmWave $\mathrm{RRH}$ approach and frequency domain $\mathrm{RoF}$, are required to enable centralized processing, while simultaneously reducing deployment cost and energy consumption.

\section{ACKNOWLEDGEMENT}

The research leading to these results has received funding from the European Union Seventh Framework Programme (FP7/2007-2013) under grant agreement no. 317941. The authors would like to acknowledge the contributions of their colleagues in iJOIN, although the views expressed are those of the authors and do not necessarily represent the project.

\section{REFERENCES}

[1] eMobility NetWorld, "Deliverable D1.1, White Paper on Broadband Wireless Beyond 2020," Jul. 2011.

[2] — - "Deliverable D1.2, Strategic Applications, Research and Innovation Agenda," Jul. 2011.

[3] C. Bernardos, A. D. Domenico, J. Ortin, P. Rost, and D. Wübben, "Challenges of designing jointly the backhaul and radio access network in a cloud-based mobile network," in Future Network \& Mobile Summit 2013, Lisbon, Portugal, Jul 2013.

[4] D. Sabella, P. Rost, Y. Sheng, E. Pateromichelakis, U. Salim, P. GuittonOuhamou, M. D. Girolamos, and G. Giuliani, "RAN as a Service: Challenges of designing a flexible RAN architecture in a cloud-based heterogeneous mobile network," in Future Network \& Mobile Summit 2013, Lisbon, Portugal, Jul 2013.

[5] P. Eriksson and B. Odenhammar, "VDSL2: Next important broadband technology," Ericsson Review No. 1, 2006.

[6] T. Orphanoudakis, E. Kosmatos, J. Angelopoulos, and A. Stavdas, "Exploiting PONs for mobile backhaul," Communications Magazine, IEEE, vol. 51, no. 2, pp. S27-S34, 2013.

[7] ITU-T, Rec. G.984.3: Gigabit-capable Passive Optical Networks ( $G$ PON): Transmission Convergence Layer Specification, ITU Std., Mar. 2008.

[8] IEEE Std 802.3ah-2004, ITU Std., 2004.

[9] "Full Service Access Network group:", http://www.fsan.org/.

[10] ITU-T, Rec. G.987.1: 10-Gigabit-capable passive optical networks (XGPON): General requirements, ITU Std., Jan. 2010.

[11] "Understanding the performance of free space optics," White Paper, LightPointe Communications Inc., 2009.

[12] J. Wells, "Faster than fiber: The future of multi-Gb/s wireless," $M i_{-}$ crowave Magazine, IEEE, vol. 10, no. 3, pp. 104-112, 2009.

[13] S. Little, "Is microwave backhaul up to the $4 \mathrm{G}$ task?" Microwave Magazine, IEEE, vol. 10, no. 5, pp. 67-74, 2009.

[14] X. Huang, Y. Guo, A. Zhang, and V. Dyadyuk, "A multi-gigabit microwave backhaul," Communications Magazine, IEEE, vol. 50, no. 3, pp. 122-129, 2012.

[15] "80G Family Data Sheet," BridgeWave Communications, Inc., 2012.

[16] G. Fettweis, F. Guderian, and S. Krone, "Entering the Path Towards Terabit/s Wireless Links," in Design, Automation Test in Europe Conference Exhibition (DATE), 2011, 2011, pp. 1-6.

[17] TR 36.932 V12.0.0 - Scenarios and requirements for small cell enhancements for E-UTRA and E-UTRAN, 3rd Generation Partnership Project Std.

[18] "AireBeam G80-2.5-MX Data Sheet," LightPointe Communications, Inc., 2013. 